June 19, 2016

\begin{abstract}
Associate Editor
International Journal of Control

Reviewing process of the manuscript:

"A Center-Free Approach for Resource Allocation with Lower Bounds"

ID: TCON-2015-1042.R1
\end{abstract}

Dear Associate Editor:

We would like to thank you for your comments and kind consideration of our manuscript. After a revision of the paper, we have properly incorporated the suggestions in the revised draft. We enclose a response report with all the reviewers' questions carefully answered. We look forward to hearing back from you.

Respectfully,

The Authors 
June 19, 2016

Responses to Reviewers' comments

Reviewing process of the manuscript:

"A Center-Free Approach for Resource Allocation with Lower Bounds"

ID: TCON-2015-1042.R1

Submitted to

International Journal of Control.

Dear Reviewers:

We would like to thank you for your insightful comments and recommendations. After this revision, the paper is more complete and clear. In this letter, we have answered to the specific comments of the reviewers.

\section{Comments of Reviewer 1}

1. The references seem outdated and should be revised. There are some papers from 2015 which are still stated as "to appear".

Answer: We have enhanced the literature review. The following references have been updated:

- E. Ramirez-Llanos and S. Martinez, "Distributed and robust resource allocation algorithms for multi-agent systems via discrete-time iterations", in Proceedings of the 54th IEEE Conference on Decision and Control, pp. 1390-1395, IEEE, 2015.

- A. Cherukuri and J. Cortés, "Distributed generator coordination for initialization and anytime optimization in economic dispatch", IEEE Transactions on Control of Network Systems, vol. 2, no. 3, pp. 226-237, 2015.

Moreover, we have included the following recent relevant references:

- H. Lee, K. J. Lee, H. Kim, B. Clerckx, and I. Lee, "Resource allocation techniques for wireless powered communication networks with energy storage constraint", IEEE Transactions on Wireless Communications, vol. 15, no. 4, pp. 2619-2628, 2016.

- J. I. Poveda and N. Quijano, "Shahshahani gradient-like extremum seeking", Automatica, vol. 58, pp. 51-59, 2015.

- M. Patriksson and C. Stromberg, "Algorithms for the continuous nonlinear resource allocation problems - new implementations and numerical studies", European Journal of Operational Research, vol. 243, no. 3, pp. 703-722, 2015.

- A. Pietrabissa, F. Delli Priscoli, A. Di Giorgio, A. Giuseppi, M. Panfili, and V. Suraci, 
"An approximate dynamic programming approach to resource management in multicloud scenarios", International Journal of Control, DOI: 10.1080/00207179.2016.1185802, 2016.

- J. Barreiro-Gomez, G. Obando, and N. Quijano, "Distributed population dynamics: Optimization and control applications", IEEE Transactions on Systems, Man, and Cybernetics: Systems, DOI: 10.1109/TSMC.2016.2523934, 2016.

- U. Ayesta, M. Erausquin, E. Ferreira, and P. Jacko, "Optimal dynamic resource allocation to prevent defaults", Operations Research Letters, vol. 44, no. 4, pp. 451-456, 2016.

- L. Tan, Z. Zhu, F. Ge, and N. Xiong, "Utility maximization resource allocation in wireless networks: Methods and algorithms", IEEE Transactions on Systems, Man, and Cybernetics: Systems, vol. 45, no. 7, pp. 1018-1034, 2015.

- T. M. Hansen, R. Roche, S. Suryanarayanan, A. A. Maciejewski, and H. J. Siegel, "Heuristic optimization for an aggregator-based resource allocation in the smart grid", IEEE Transactions on Smart Grid, vol. 6, no. 4, pp. 1785-1794, 2015.

- P. S. Pillai and S. Rao, "Resource allocation in cloud computing using the uncertainty principle of game theory", IEEE Systems Journal, vol. 10, no. 2, pp. 637-648, 2016.

2. The motivating example is interesting even tough it is limited to a plant with 7 chillers. The fact that the example is small is acceptable but the motivating application should describe at least the prospect of a large-scale application to motivate distributed optimization on an arbitrary graph (and thus autonomous decision making by the devices). Nevertheless, the proposed method seems indeed applicable to other large-scale scenarios.

Answer: The applications section (Section VI in the article) has been modified. On the one hand, we have improved the motivation of using the distributed algorithm proposed in our manuscript in problems involving air conditioning systems that have multiple chillers. Basically, the motivation lies in two points. First, the objective of describing this small-scale application is to allow the readers to grasp the essence of the proposed approach and understand the efficacy of the resulting solutions. Second, small-scale systems can also benefit from using distributed approaches because these approaches increase systems resilience in the face of central failures. In the manuscript, the motivation of considering multiple chiller problems reads as follows:

"First, we present an application for sharing load in multiple chillers plants. Although this is not a large-scale application (multi-chiller plants are typically comprised of less than ten chillers [36]), it aims to illustrate the essence of the proposed method and shows algorithm's performance in small size-problems. One of the reasons to use a distributed approach in small/medium-size systems is due to the need of enhancing systems resilience in the face of central failures (e.g., in multiple chiller plants, central failures can occur due to cyber-attacks [37] against building management systems [36])." (beginning of Section VI, pp.20).

On the other hand, we have included an additional application related to the distributed 
computation of the Euclidean projection. This application, which is described and motivated in Section VI-B, is used to illustrate the performance of the proposed algorithm in large-scale problems.

Thank you very much for your time and consideration.

Respectfully,

The Authors 